\title{
Optimal reinsurance and investment in a jump-diffusion financial market with common shock dependence
}

\author{
Zhibin Liang ${ }^{1},{ }^{*}$ Kam Chuen Yuen ${ }^{2}, \quad$ Caibin Zhang ${ }^{1}$ \\ ${ }^{1}$ School of Mathematical Sciences and Institute of Finance and Statistics, \\ Nanjing Normal University, Jiangsu 210023, P.R.China \\ 2 Department of Statistics and Actuarial Science, \\ The University of Hong Kong, Pokfulam Road, Hong Kong, P.R.China
}

\begin{abstract}
In this paper, we study the optimal reinsurance and investment problem in a financial market with jump-diffusion risky asset. It is assumed that the insurance risk model is modulated by a compound Poisson process, and that the jumps in both the risky asset and insurance risk process are correlated through a common shock. Under the criterion of maximizing the expected exponential utility, we adopt a nonstandard approach to examine the existence and uniqueness of the optimal strategy. Using the technique of stochastic control theory, closed-form expressions for the optimal strategy and the value function are derived not only for the expected value principle but also for the variance premium principle. Also, we investigate the effect of the common shock parameter as well as some other important parameters on the optimal strategies. In particular, a numerical example shows that the optimal investment strategy decreases as the degree of common shock dependence increases but the optimal insurance retention level does not behave the same.
\end{abstract}

Keywords: Exponential utility; Hamilton-Jacobi-Bellman equation; Common shock dependence; Investment/reinsurance; Jump-diffusion process

AMS 2000 Subject Classification: 91B28, 91B30, 93E20.

\footnotetext{
*Corresponding author: Dr. Zhibin Liang, Email: liangzhibin111@hotmail.com
} 


\section{Introduction}

In recent years, optimal reinsurance and investment problems for various risk models have gained a lot of interest in the financial and actuarial literatures. The technique of stochastic control theory and the corresponding Hamilton-Jacobi-Bellman equation are frequently used to cope with these optimization problems. See, for example, Schmidli (2002), Irgens and Paulsen (2004), Promislow and Young (2005), Bai and Guo (2008), Luo et al. (2008), Liang et al. (2011), Liang et al. (2012), Liang and Bayraktar (2014), Bi et al.(2016), Landriault et al. (2016), Zheng et al. (2016).

With reinsurance, insurers are able to transfer some of their risks to another party at the expense of making less potential profit, and hence finding optimal reinsurance strategy to balance their risk and profit is of great interest to them. In the study of optimal reinsurance contracts, a few objective functions are commonly seen in the literature. Browne (1995), Schmidli (2001), Liang (2007), and Luo et al. (2008) consider the objective function that minimizes ruin probability. Kaluszka (2001, 2004) study the optimal reinsurance problem under various mean-variance premium principles of the reinsurer. Since explicit expression for ruin probability is difficult to derive when the underlying risk follows a compound Poisson process, some papers including Centeno (1986, 2002), Hald and Schmidli (2004), and Liang and Guo (2007, 2008) focus on constructing optimal contracts that maximize the adjustment coefficient by the martingale approach. Moreover, Cai and Tan (2007), Cai et al. (2008), and Bernard and Tian (2009) adopt the criteria of minimizing tail risk measures such as value at risk and conditional tail expectation. Our objective in this paper is to maximize the expected utility of terminal wealth, which is another popular criterion for various optimization problems in finance and modern risk theory. See, for example, Irgens and Paulsen (2004), Zhang and Yang (2005), Liang et al. (2011), Yuen et al. (2015), Zhang et al. (2016), and the references therein.

Besides reinsurance, investment in some risky assets is another important source of risk control. Most of the literature on investment optimization is based on the assumption that stock price follows a diffusion-type process, in particular a geometric Brownian motion. However, in the real financial market, information often comes as a surprise. Such information usually leads to a jump in the price of stock. Therefore, to model stock price, one may consider using a jump-diffusion model which allow stock price to jump to a new level and to follow a geometric Brownian motion right after discrete jumps.

In this paper, under the criterion of maximizing the expected utility of terminal wealth, we study both optimal reinsurance which is constrained to the interval $[0,1]$ and optimal investment with a jump-diffusion risky asset and no short selling. Moreover, the aggregate claims and the stock price are correlated by means of a common shock. This kind of model assumes that there exists a common shock affecting the stock market as well as the insurance market. In reality, a common component can depict a big event which has negative impact on both the financial and insurance markets. For example, severe events like earthquakes or prolonged extreme weathers not only has 
immediate adverse impact on financial markets, but also induce various insurance claims. Similar argument applies to other detrimental incidents caused by human or natural disasters. Hence, we generalize the model of $\mathrm{Bi}$ and Guo (2013) from the independent setting to the case where the aggregate claims process and risky asset process are dependent. We also extend the assumption of negative jump in Alvarez E. et al. (2014) to negative expected value of jump, which allows the inclusion of positive jumps, and at the same time also meets the observed fact that the stock market reactions to negative shocks are usually significantly stronger than the reactions to positive ones. Therefore, the model of study is practically more reasonable, and definitely makes the optimization problem more complicated and challenging.

Although research on optimal reinsurance and investment is increasing rapidly, very few of them deal with the problem in relation to dependence between the risky asset and insurance aggregate claims. Within the mean-variance framework, Liang et al. (2016) study the optimal reinsurance and investment problem in a financial market with jump-diffusion risky asset, where the insurance risk model is modulated by a compound Poisson process, and the two jump-number processes are correlated through a common shock. This paper extends their work to the optimization problem of maximizing the expected utility of terminal wealth with the constraint that the optimal reinsurance strategy lies in the unit interval. Using the technique of stochastic control theory and the corresponding Hamilton-Jacobi-Bellman equation, we adopt a nonstandard approach to examine the existence and uniqueness of the optimal strategy, and derive the closed-form expressions for the optimal strategy and the value function not only for the expected value principle but also for the variance premium principle. Also, we investigate the effect of the common shock parameter as well as some other important parameters on the optimal strategies.

The rest of the paper is organized as follows. In Section 2, the model and assumptions are presented. The main results and the closed-form expressions for the optimal values are derived in Sections 3. In Sections 4 and 5, we investigate the effects of the common shock as well as other model parameters on the optimal strategy. Some numerical examples are presented to illustrate the impact of the parameters on the optimal strategy in Section 6. Finally, Section 7 concludes the paper.

\section{Model and problem formulation}

Let $(\Omega, \mathcal{F}, P)$ be a probability space with filtration $\left\{\mathcal{F}_{t}\right\}$ containing all objects defined in the following.

We consider the financial market where the assets are traded continuously on a finite time horizon $[0, T]$. There are a risk-free asset (bond) and a risky asset (stock) in the financial market. The 
price of the bond is given by

$$
\left\{\begin{aligned}
d B(t) & =r B(t) d t, \quad t \in[0, T], \\
B(0) & =1,
\end{aligned}\right.
$$

where $r(>0)$ is the interest rate of the bond, and it is a constant.

The price of the stock is modeled by the following jump-diffusion process

$$
\left\{\begin{array}{l}
d S(t)=S(t-)\left[b(t) d t+\sigma(t) d W(t)+d \sum_{i=1}^{M_{2}(t)} Y_{i}\right], \quad t \in[0, T], \\
S(0)=S_{0}
\end{array}\right.
$$

where $S_{0}$ is the deterministic initial price, $b(t)(>r)$ is the appreciation rate, $\sigma(t)>0$ is the volatility coefficient, and $\{W(t)\}_{t \geq 0}$ is a standard $\left\{\mathcal{F}_{t}\right\}_{t \geq 0}$-adapted Brownian motion, $\left\{M_{2}(t)\right\}_{t \geq 0}$ is a Poisson process with intensity parameter $\lambda_{2}+\lambda>0$, and the jump sizes $\left\{Y_{i}, i \geq 1\right\}$ are assumed to be an i.i.d sequence with values in $(-1, \infty)$. It is assumed that $b(t)$ and $\sigma(t)$ are deterministic, Borelmeasurable and bounded on $[0, T]$. Note that $Y_{i}>-1$ ensures the positivity of the stock price. Let $Y$ be a generic random variable which has the same distribution as $Y_{i}, i \geq 1$, and $F_{Y}(\cdot)$ be the cumulative distribution function of $Y$. We assume that $E(Y)=\mu_{21}$ and $E\left(Y^{2}\right)=\mu_{22}>0$, the moment generating function $M_{Y}(r)$ exists, and that $\{W(t)\}_{t \geq 0},\left\{M_{2}(t)\right\}_{t \geq 0}$ and $\left\{Y_{i}, i \geq 1\right\}$ are mutually independent. Furthermore, we impose throughout this paper the following assumption.

Assumption 2.1. The value of the expected jump size $\mu_{21}$ satisfies

$$
-\frac{a(t)}{\lambda+\lambda_{2}}<\mu_{21}<0
$$

where $a(t)=b(t)-r>0$.

The assumption of $\mu_{21}<0$ mainly comes from an empirically observed fact that the stock market reactions to negative shocks are usually significantly stronger than the reactions to positive ones (see Alvarez E et al. (2014)). This is also the celebrated "bad news" principle originally introduced in the seminal study by Bernanke (1983). Moreover, in the economic market, the expected return rate of the risky asset would be larger than the risk-free interest rate. Therefore, it is reasonable to assume that $b(t)+\left(\lambda_{2}+\lambda\right) \mu_{21}>r$, which can be rewritten as $a(t)+\left(\lambda_{2}+\lambda\right) \mu_{21}>0$, or equivalently, $\mu_{21}>-a(t) /\left(\lambda_{2}+\lambda\right)$.

The diffusion component in equation (2.1) characterizes the normal fluctuation in the stock price, due to gradual changes in economic conditions or the arrival of new information which causes marginal changes in the stock price. The jump component describes sudden changes in the stock price due to the arrival of important new information which has a large effect on the stock price. By stochastic differential equation (SDE) theory, a unique solution exists for SDE (2.1). 
The risk process $\{U(t)\}_{t \geq 0}$ of the insurer is modeled by

$$
d U(t)=c d t-d \sum_{i=1}^{M_{1}(t)} X_{i}, \quad U(0)=U_{0},
$$

where $U_{0}$ is the deterministic initial reserve of the insurer, the constant $c$ is the premium rate, the claim-number process $\left\{M_{1}(t)\right\}_{t \geq 0}$ is a Poisson process with intensity $\lambda_{1}+\lambda>0, X_{i}$ is the size of the $i$ th claim, and $\left\{X_{i}, i \geq 1\right\}$ are assumed to be an i.i.d. sequence and independent of $\left\{M_{1}(t)\right\}_{t \geq 0}$. Note that the compound Poisson process $\sum_{i=1}^{M_{1}(t)} X_{i}$ represents the cumulative amount of claims in time interval $[0, t]$. Let $X$ be a generic random variable which has the same distribution as $X_{i}, i \geq 1$, and $F_{X}(\cdot)$ be the cumulative distribution function of $X$. Write the mean of $X$ and the second moment of $X$ as $E(X)=\mu_{11}>0$ and $E\left(X^{2}\right)=\mu_{12}>0$, respectively, and assume that the moment generating function $M_{X}(r)$ exists. The risk process defined in equation (2.2), from the perspective of the insurer, is really a pay-off process associated with the (insurance) contracts he (or she) has entered. Furthermore, the two jump processes $\left\{M_{1}(t)\right\}_{t \geq 0}$ and $\left\{M_{2}(t)\right\}_{t \geq 0}$ are correlated in the way that

$$
M_{1}(t)=N_{1}(t)+N(t) \quad \text { and } \quad M_{2}(t)=N_{2}(t)+N(t),
$$

with $N_{1}(t), N_{2}(t)$, and $N(t)$ being three independent Poisson processes with parameters $\lambda_{1}, \lambda_{2}$, and $\lambda$, respectively. It is obvious that the dependence between the financial risky asset and the aggregate claims processes is due to a common shock governed by the counting process $N(t)$. Moreover, it is assumed that $\{W(t)\}_{t \geq 0},\left\{N_{1}(t)\right\}_{t \geq 0},\left\{X_{i}, i \geq 1\right\},\left\{N_{2}(t)\right\}_{t \geq 0},\left\{Y_{i}, i \geq 1\right\}$ and $\{N(t)\}_{t \geq 0}$ are mutually independent.

Assume that the insurer is allowed to invest all of his (or her) wealth in the financial market. Let $\xi(t)$ and $u(t)$ denote the total market value of the agent's wealth in the bond and stock, respectively, and $\xi(t)+u(t)=R(t)$. In this paper, short-selling of the stock is prohibited, i.e., $u(t) \geq 0$, but $\xi(t)$ is not constrained. Furthermore, it is assumed that the insurance company can purchase proportional reinsurance. That is, it can reinsure a fraction of its claims with the retention proportion $q(t) \in[0,1]$ for a claim occurring at time $t$. As a result, the insurer pays $q(t) X$ of a claim occurring at time $t$, and the reinsurer pays $(1-q(t)) X$. Also, the reinsurance premium is paid at rate $\delta(q(t))$. A strategy $\pi(t)=(u(t), q(t))$ is said to be admissible if $u(t)$ and $q(t)$ are $\mathcal{F}_{t}$-predictable processes, and satisfy $u(t) \geq 0, q(t) \in[0,1]$, and $E\left[\int_{0}^{t} u^{2}(s) d s\right]<\infty$ for all $t \geq 0$. We denote the set of all admissible strategies by $\Pi$. Then the resulting surplus process $R(t)$ is given by

$$
\left\{\begin{aligned}
d R(t)= & {[r R(t)+a(t) u(t)+c-\delta(q(t))] d t+u(t) \sigma(t) d W(t) } \\
& +u(t) d \sum_{i=1}^{M_{2}(t)} Y_{i}-q(t) d \sum_{i=1}^{M_{1}(t)} X_{i} \\
R(0)= & R_{0} .
\end{aligned}\right.
$$


Corresponding to an admissible trading strategy $\pi(\cdot)$ and a deterministic initial capital $R_{0}$, there exists a unique $R(t)$ satisfies (2.3).

Suppose that the insurer is interested in maximizing the expected utility of terminal wealth, say at time $T$. The utility function is $w(x)$, which satisfies $w^{\prime}>0$ and $w^{\prime \prime}<0$. Then the objective function is

$$
J^{\pi}(t, x)=E[w(R(T)) \mid R(t)=x],
$$

and the corresponding value function is given by

$$
V(t, x)=\sup _{\pi \in \Pi} J^{\pi}(t, x)
$$

We assume that the insurer has an exponential utility function

$$
w(x)=-\frac{\beta}{\nu} e^{-\nu x}
$$

with $\beta>0$ and $\nu>0$. This utility has constant absolute risk aversion (CARA) parameter $\nu$. Such a utility function plays an important role in insurance mathematics and actuarial practice as this is the only function under which the principle of "zero utility" gives a fair premium that is independent of the level of reserve of an insurance company (see Gerber (1979)).

Let $C^{1,2}$ denote the space of $\phi(t, x)$ such that $\phi$ and its partial derivatives $\phi_{t}, \phi_{x}$ and $\phi_{x x}$ are continuous on $[0, T] \times \mathbb{R}$. To solve the optimization problem, we use the dynamic programming approach described in Fleming and Soner (2006). From the standard arguments, we see that if the value function $V \in C^{1,2}$, then $V$ satisfies the following Hamilton-Jacobi-Bellman (HJB) equation

$$
\sup _{\pi \in \Pi} \mathcal{A}^{\pi} V(t, x)=0
$$

for $t<T$ with the boundary condition

$$
V(T, x)=w(x)
$$

where

$$
\begin{aligned}
\mathcal{A}^{\pi} V(t, x)= & V_{t}(t, x)+[r x+a(t) u+c-\delta(q)] V_{x}(t, x)+\frac{1}{2} \sigma(t)^{2} u^{2} V_{x x}(t, x) \\
& +\lambda_{2} E[V(t, x+u Y)-V(t, x)]+\lambda_{1} E[V(t, x-q X)-V(t, x)] \\
& +\lambda E[V(t, x+u Y-q X)-V(t, x)] .
\end{aligned}
$$

Using the standard methods of Fleming and Soner (2006), we have the following verification theorem.

Theorem 2.1. Let $W \in C^{1,2}$ be a classical solution to (2.5) that satisfies (2.6). Then the value function $V$ given by (2.4) coincides with $W$. That is,

$$
W(t, x)=V(t, x)
$$


Furthermore, set $\pi^{*}=\left(u^{*}, q^{*}\right)$ such that

$$
\mathcal{A}^{\pi^{*}} V(t, x)=0
$$

holds for all $(t, x) \in[0, T) \times \mathbb{R}$. Then $\pi^{*}\left(t, R^{*}(t)\right)=\left(u^{*}\left(t, R^{*}(t)\right), q^{*}\left(t, R^{*}(t)\right)\right)$ is the optimal strategy, where $R^{*}(t)$ is the surplus process under the optimal strategy.

\section{Optimal results for the compound Poisson risk model}

In this section, we consider the optimization problem for risk model (2.3). To solve the equation

$$
\begin{aligned}
\sup _{u, q} & \left\{V_{t}(t, x)+[r x+a(t) u+c-\delta(q)] V_{x}(t, x)+\frac{1}{2} \sigma(t)^{2} u^{2} V_{x x}(t, x)\right. \\
& +\lambda_{2} E[V(t, x+u Y)-V(t, x)]+\lambda_{1} E[V(t, x-q X)-V(t, x)] \\
& +\lambda E[V(t, x+u Y-q X)-V(t, x)]\}=0,
\end{aligned}
$$

with the boundary condition $V(T, x)=w(x)$, inspired by Browne (1995), we try to fit a solution of the form

$$
V(t, x)=-\frac{\beta}{\nu} \exp \left[-\nu x e^{r(T-t)}+h(T-t)\right]
$$

where $h(\cdot)$ is a suitable function such that (3.1) is a solution to (2.5). The boundary condition $V(T, x)=w(x)$ implies that $h(0)=0$.

From (3.1), we get

$$
\left\{\begin{array}{l}
V_{t}=V(t, x)\left[\nu x r e^{r(T-t)}-h^{\prime}(T-t)\right], \\
V_{x}=V(t, x)\left[-\nu e^{r(T-t)}\right], \\
V_{x x}=V(t, x)\left[\nu^{2} e^{2 r(T-t)}\right], \\
E[V(t, x-q X)-V(t, x)]=V(t, x)\left[M_{X}\left(\nu q e^{r(T-t)}\right)-1\right], \\
E[V(t, x+u Y)-V(t, x)]=V(t, x)\left[M_{Y}\left(-\nu u e^{r(T-t)}\right)-1\right], \\
E[V(t, x+u Y-q X)-V(t, x)]=V(t, x)\left[M_{X}\left(\nu q e^{r(T-t)}\right) M_{Y}\left(-\nu u e^{r(T-t)}\right)-1\right] .
\end{array}\right.
$$

Putting (3.2) back into equation (2.5) and canceling the like terms yield

$$
\begin{aligned}
\inf _{u, q}\{ & -h^{\prime}(T-t)-(u a(t)+c-\delta(q)) \nu e^{r(T-t)}+\frac{1}{2} u^{2} \sigma^{2}(t) \nu^{2} e^{2 r(T-t)} \\
& +\lambda_{1}\left[M_{X}\left(\nu q e^{r(T-t)}\right)-1\right]+\lambda_{2}\left[M_{Y}\left(-\nu u e^{r(T-t)}\right)-1\right] \\
& \left.+\lambda\left[M_{X}\left(\nu q e^{r(T-t)}\right) \cdot M_{Y}\left(-\nu u e^{r(T-t)}\right)-1\right]\right\}=0,
\end{aligned}
$$


for $t<T$. Let

$$
\begin{aligned}
f(u, q)= & (\delta(q)-u a(t)) \nu e^{r(T-t)}+\frac{1}{2} u^{2} \sigma^{2}(t) \nu^{2} e^{2 r(T-t)} \\
& +\lambda_{1} M_{X}\left(\nu q e^{r(T-t)}\right)+\lambda_{2} M_{Y}\left(-\nu u e^{r(T-t)}\right) \\
& +\lambda M_{X}\left(\nu q e^{r(T-t)}\right) \cdot M_{Y}\left(-\nu u e^{r(T-t)}\right) .
\end{aligned}
$$

For any $t \in[0, T]$, we have

$$
\left\{\begin{array}{l}
\frac{\partial f(u, q)}{\partial u}=\left[-a(t)+u \sigma^{2}(t) \nu e^{r(T-t)}-M_{Y}^{\prime}\left(-\nu u e^{r(T-t)}\right)\left(\lambda_{2}+\lambda M_{X}\left(\nu q e^{r(T-t)}\right)\right)\right] \cdot \nu e^{r(T-t)}, \\
\frac{\partial f(u, q)}{\partial q}=\left[\delta^{\prime}(q)+M_{X}^{\prime}\left(\nu q e^{r(T-t)}\right)\left(\lambda_{1}+\lambda M_{Y}\left(-\nu u e^{r(T-t)}\right)\right)\right] \cdot \nu e^{r(T-t)}, \\
\frac{\partial^{2} f(u, q)}{\partial u^{2}}=\left[\sigma^{2}(t)+M_{Y}^{\prime \prime}\left(-\nu u e^{r(T-t)}\right)\left(\lambda_{2}+\lambda M_{X}\left(\nu q e^{r(T-t)}\right)\right)\right] \cdot \nu^{2} e^{2 r(T-t)}>0, \\
\frac{\partial^{2} f(u, q)}{\partial q^{2}}=\delta^{\prime \prime}(q) \nu e^{r(T-t)}+M_{X}^{\prime \prime}\left(\nu q e^{r(T-t)}\right)\left(\lambda_{1}+\lambda M_{Y}\left(-\nu u e^{r(T-t)}\right)\right) \cdot \nu^{2} e^{2 r(T-t)}, \\
\frac{\partial^{2} f(u, q)}{\partial u \partial q}=-\lambda M_{X}^{\prime}\left(\nu q e^{r(T-t)}\right) M_{Y}^{\prime}\left(-\nu u e^{r(T-t)}\right) \cdot \nu^{2} e^{2 r(T-t)},
\end{array}\right.
$$

where $M_{Z}^{\prime}(r)=E\left(Z e^{r Z}\right)$ and $M_{Z}^{\prime \prime}(r)=E\left(Z^{2} e^{r Z}\right)$.

Lemma 3.1. The function $f(u, q)$ is a convex function with respect to $u$ and $q$, respectively.

Proof. To prove $f(u, q)$ is a convex function with respect to (w.r.t.) $u$ and $q$, it is sufficient to prove the Hessian matrix of $f(u, q)$ is a positive definite matrix.

Let matrices

$$
\mathbf{A}=\left(\begin{array}{cc}
\sigma^{2}(t)+\lambda_{2} M_{Y}^{\prime \prime}(\cdot) & 0 \\
0 & \delta^{\prime \prime}(q) \nu^{-1} e^{-r(T-t)}+\lambda_{1} M_{X}^{\prime \prime}(\cdot)
\end{array}\right)
$$

and

$$
\mathbf{B}=\left(\begin{array}{cc}
M_{X}(\cdot) M_{Y}^{\prime \prime}(\cdot) & -M_{X}^{\prime}(\cdot) M_{Y}^{\prime}(\cdot) \\
-M_{X}^{\prime}(\cdot) M_{Y}^{\prime}(\cdot) & M_{X}^{\prime \prime}(\cdot) M_{Y}(\cdot)
\end{array}\right) .
$$

Then the Hessian matrix can be decomposed as

$$
\left(\begin{array}{cc}
\frac{\partial^{2} f(u, q)}{\partial u^{2}} & \frac{\partial^{2} f(u, q)}{\partial u \partial q} \\
\frac{\partial^{2} f(u, q)}{\partial q \partial u} & \frac{\partial^{2} f(u, q)}{\partial q^{2}}
\end{array}\right)=(\mathbf{A}+\lambda \cdot \mathbf{B}) \cdot \nu^{2} e^{2 r(T-t)} .
$$

It is easy to see that matrix $\mathbf{A}$ is a positive definite matrix. Also, it follows from the Cauchy-Schwarz inequality that matrix $\mathbf{B}$ is a nonnegative definite matrix. Thus, we can conclude that the Hessian matrix is positive definite. 
Therefore, the minimizer $(\bar{u}, \bar{q})$ of $f(u, q)$ satisfies the equations

$$
\left\{\begin{array}{l}
-a(t)+u \sigma^{2}(t) \nu e^{r(T-t)}-M_{Y}^{\prime}\left(-\nu u e^{r(T-t)}\right)\left(\lambda_{2}+\lambda M_{X}\left(\nu q e^{r(T-t)}\right)\right)=0, \\
\delta^{\prime}(q)+M_{X}^{\prime}\left(\nu q e^{r(T-t)}\right)\left(\lambda_{1}+\lambda M_{Y}\left(-\nu u e^{r(T-t)}\right)\right)=0 .
\end{array}\right.
$$

Moreover, using arguments similar to those in the proof of Lemma 3.2 of Yuen et al. (2015), one can derive the following result.

Lemma 3.2. For any $t \in[0, T]$, if both $\hat{\pi}=(\hat{u}, \hat{q})$ and $\tilde{\pi}=(\tilde{u}, \tilde{q})$ are the solutions to equation (3.4), then we have $\hat{\pi}=\tilde{\pi}$.

Then the uniqueness of the solution to equation (3.4) can be proved using Lemma 3.2. The following two subsections discuss the optimal investment and reinsurance problem in details for two kinds of premium principle: expected value principle and variance premium principle.

\subsection{Expected value principle}

In this subsection, we assume that the reinsurance premium is calculated according to the expected value principle. That is,

$$
\delta(q)=(1+\eta)(1-q) a_{1},
$$

where $a_{1}=\left(\lambda_{1}+\lambda\right) \mu_{11}$ and $\eta$ is the safety loading of the reinsurer. Then equation (3.4) becomes

$$
\left\{\begin{array}{l}
-a(t)+u \sigma^{2}(t) \nu e^{r(T-t)}-M_{Y}^{\prime}\left(-\nu u e^{r(T-t)}\right)\left(\lambda_{2}+\lambda M_{X}\left(\nu q e^{r(T-t)}\right)\right)=0 \\
-(1+\eta) a_{1}+M_{X}^{\prime}\left(\nu q e^{r(T-t)}\right)\left(\lambda_{1}+\lambda M_{Y}\left(-\nu u e^{r(T-t)}\right)\right)=0
\end{array}\right.
$$

or equivalently,

$$
\left\{\begin{array}{l}
a(t)+\sigma^{2}(t) n+M_{Y}^{\prime}(n)\left(\lambda_{2}+\lambda M_{X}(m)\right)=0 \\
-(1+\eta) a_{1}+M_{X}^{\prime}(m)\left(\lambda_{1}+\lambda M_{Y}(n)\right)=0
\end{array}\right.
$$

where $n=-\nu u e^{r(T-t)}$ and $m=\nu q e^{r(T-t)}$, and we have

Lemma 3.3. Under Assumption 2.1, there is a unique solution to each of the following equations

$$
a(t)+\mu_{21}\left(\lambda_{2}+\lambda M_{X}(m)\right)=0,
$$

and

$$
a(t)+\sigma^{2}(t) n+M_{Y}^{\prime}(n)\left(\lambda_{2}+\lambda\right)=0,
$$

for any $t \in[0, T]$. 
Proof. Under Assumption 2.1, we can directly obtain the unique positive solution to equation (3.6) given by

$$
m_{1}(t)=M_{X}^{-1}\left(-\frac{a(t)+\lambda_{2} \mu_{21}}{\lambda \mu_{21}}\right) .
$$

We now consider equation (3.7). Let

$$
g_{1}(n)=a(t)+\sigma^{2}(t) n \quad \text { and } \quad g_{2}(n)=-M_{Y}^{\prime}(n)\left(\lambda_{2}+\lambda\right) .
$$

Then we have

$$
\begin{aligned}
& g_{2}(0)=-\left(\lambda_{2}+\lambda\right) \mu_{21}, \\
& g_{2}^{\prime}(n)=-\left(\lambda_{2}+\lambda\right) M_{Y}^{\prime \prime}(n)<0 .
\end{aligned}
$$

That is, for any $t \in[0, T], g_{2}(n)$ is a strictly decreasing function with $g_{2}(0)=-\left(\lambda_{2}+\lambda\right) \mu_{21}$. Furthermore, $g_{1}(n)$ is an increasing linear function with

$$
g_{1}(0)=a(t)>g_{2}(0) .
$$

Therefore, $g_{1}(n)$ and $g_{2}(n)$ have a unique point of intersection at some $n_{1}(t)<0$. That is, equation (3.7) has a unique negative root.

Lemma 3.4. For any $t \in[0, T]$, the unique positive solution to the equation

$$
-(1+\eta) a_{1}+M_{X}^{\prime}(m)\left(\lambda_{1}+\lambda\right)=0
$$

is

$$
m_{2}=\left(M_{X}^{\prime}\right)^{-1}\left((1+\eta) \mu_{11}\right) .
$$

Furthermore, there exists a unique negative root $n_{2}<0$ of the equation

$$
-(1+\eta) a_{1}+\mu_{11}\left(\lambda_{1}+\lambda M_{Y}(n)\right)=0 .
$$

Proof. For equation (3.8), simple calculations yield $m_{2}$. We now consider equation (3.9), which can be rewritten as

$$
M_{Y}(n)=1+\frac{\lambda_{1}+\lambda}{\lambda} \cdot \eta .
$$

Denote $g(n)=M_{Y}(n)$. So, we have $g^{\prime}(n)=M_{Y}^{\prime}(n)=E\left(Y e^{n Y}\right)$ and

$$
g^{\prime \prime}(n)=E\left(Y^{2} e^{n Y}\right)>0 .
$$

Therefore, $g^{\prime}(n)$ is a strictly increasing function w.r.t. $n$, and thus for any $n \leq 0$, we have $g^{\prime}(n) \leq$ $g^{\prime}(0)=E(Y)=\mu_{21}<0$. If we have $g^{\prime}(n)>0$ for some $n>0$, then there exists an $n_{0}>0$ such that $g^{\prime}\left(n_{0}\right)=0$, and thus we have $g^{\prime}(n)<0$ for any $n<n_{0}$; on the other hand, for any $n>n_{0}$, we have $g^{\prime}(n)>0$, which means that function $g(n)$ is a decreasing convex function w.r.t. $n \leq n_{0}$. 
Since $g(0)=M_{Y}(0)=1$, there exists a unique $n_{2}<0$ such that $g\left(n_{2}\right)=1+\left(\left(\lambda_{1}+\lambda\right) / \lambda\right) \cdot \eta$. That is, equation

$$
M_{Y}(n)=1+\frac{\lambda_{1}+\lambda}{\lambda} \cdot \eta
$$

has a unique negative solution. This completes the proof.

The next lemma states the existence and uniqueness of the solution to equation (3.5).

Lemma 3.5. Let $m_{1}(t)$ and $m_{2}$ be the unique positive root of equations (3.6) and (3.8), $n_{1}(t)$ and $n_{2}$ be the unique negative roots of equations (3.7) and (3.9), respectively. If inequalities

$$
\left\{\begin{array}{l}
m_{1}(t)>m_{2}, \\
n_{1}(t)>n_{2},
\end{array}\right.
$$

or

$$
\left\{\begin{array}{l}
m_{1}(t)<m_{2}, \\
n_{1}(t)<n_{2},
\end{array}\right.
$$

hold for any $t \in[0, T]$, then equation (3.5) has a unique negative root $\bar{n}(T-t)$ and positive root $\bar{m}(T-t)$.

Proof. Let

$$
H_{1}(n, m)=a(t)+\sigma^{2}(t) n+M_{Y}^{\prime}(n)\left(\lambda_{2}+\lambda M_{X}(m)\right),
$$

and assume that the equation $H_{1}(n, m)=0$ implies the relationship between $n$ and $m$ by $m=f_{1}(n)$. Under Assumption 2.1, it follows from Lemma 3.3 that the equations $H_{1}(0, m)=0$ and $H_{1}(n, 0)=0$ have unique positive solution $m_{1}(t)$ and negative solution $n_{1}(t)$, respectively. That is, for any $t \in[0, T]$, the continuous function $f_{1}(n)$ has the value of $f_{1}(0)=m_{1}(t)>0$, and $f_{1}\left(n_{1}(t)\right)=0$ with $n_{1}(t)<0$. Let

$$
H_{2}(n, m)=-(1+\eta) a_{1}+M_{X}^{\prime}(m)\left(\lambda_{1}+\lambda M_{Y}(n)\right),
$$

and assume that the equation $H_{2}(n, m)=0$ implies another relationship between $n$ and $m$ by $m=f_{2}(n)$. Along the same lines, one can show that for any $t \in[0, T]$, the continuous function $f_{2}(n)$ has the value of $f_{2}(0)=m_{2}$ and $f_{2}\left(n_{2}\right)=0$ with $m_{2}>0$ and $n_{2}<0$.

Moreover, differentiating both side of the equations $H_{1}(n, m)=0$ and $H_{2}(n, m)=0$ w.r.t. $n$ yields

$$
f_{1}^{\prime}(n)=-\frac{\sigma^{2}(t)+M_{Y}^{\prime \prime}(n)\left(\lambda_{2}+\lambda M_{X}(m)\right)}{\lambda M_{X}^{\prime}(m) \cdot M_{Y}^{\prime}(n)},
$$

and

$$
f_{2}^{\prime}(n)=-\frac{\lambda M_{X}^{\prime}(m) \cdot M_{Y}^{\prime}(n)}{M_{X}^{\prime \prime}(m)\left(\lambda_{1}+\lambda M_{Y}(n)\right)},
$$


respectively. Parallel to the proof of Lemma 3.4, it can be shown that $f_{1}(n)$ is an increasing function w.r.t. $n \leq n_{0}$, and $f_{2}(n)$ is also an increasing function w.r.t. $n \leq n_{0}$. Therefore, for any $t \in[0, T]$, if the following inequalities

or

$$
\left\{\begin{array}{l}
m_{1}(t)>m_{2}, \\
n_{1}(t) \quad>n_{2},
\end{array}\right.
$$

$$
\left\{\begin{array}{l}
m_{1}(t)<m_{2}, \\
n_{1}(t)<n_{2},
\end{array}\right.
$$

hold, the functions $f_{1}(n)$ and $f_{2}(n)$ have at least one point of intersection at some $\bar{n}(T-t)<0$. It follows from Lemma 3.2 that equation (3.5) has a unique root $(\bar{n}(T-t), \bar{m}(T-t))$ with $\bar{m}(T-t)=$ $f_{1}(\bar{n}(T-t))=f_{2}(\bar{n}(T-t))>0$.

It follows from Lemma 3.5 that $-\nu \bar{u}(T-t) e^{r(T-t)}=\bar{n}(T-t)$ and $\nu \bar{q}(T-t) e^{r(T-t)}=\bar{m}(T-t)$. Thus, we have

$$
\left\{\begin{array}{l}
\bar{u}(T-t)=-\frac{\bar{n}(T-t)}{\nu} e^{-r(T-t)} \\
\bar{q}(T-t)=\frac{\bar{m}(T-t)}{\nu} e^{-r(T-t)}
\end{array}\right.
$$

For convenient discussion, let $a(t) \equiv a$ and $\sigma(t) \equiv \sigma$ be constant parameters. Then $\bar{m}(T-t)$ and $\bar{n}(T-t)$ in Lemma 3.5 become constants $\bar{m}$ and $\bar{n}$, respectively, and

$$
m_{1}(t) \equiv m_{1}=M_{X}^{-1}\left(-\frac{a+\lambda_{2} \mu_{21}}{\lambda \mu_{21}}\right)
$$

is also a constant. Before discussing the optimal values in details, we give the following lemma.

Lemma 3.6. Under Assumption 2.1, for any $t \in[0, T]$, there exists a unique root to the equation

$$
a+\sigma^{2} n+M_{Y}^{\prime}(n)\left(\lambda_{2}+\lambda M_{X}\left(\nu e^{r(T-t)}\right)\right)=0 .
$$

Proof. Let

$$
\hat{g}_{1}(n)=a+\sigma^{2} n,
$$

and

$$
\hat{g}_{2}(n)=-M_{Y}^{\prime}(n)\left(\lambda_{2}+\lambda M_{X}\left(\nu e^{r(T-t)}\right)\right)
$$

Then we have

$$
\begin{aligned}
& \hat{g}_{2}(0)=-\left(\lambda_{2}+\lambda M_{X}\left(\nu e^{r(T-t)}\right)\right) \mu_{21}, \\
& \hat{g}_{2}^{\prime}(n)=-\left(\lambda_{2}+\lambda M_{X}\left(\nu e^{r(T-t)}\right)\right) M_{Y}^{\prime \prime}(n)<0 .
\end{aligned}
$$


That is, for any $t \in[0, T], \hat{g}_{2}(n)$ is a strictly decreasing function with

$$
\hat{g}_{2}(0)=-\left(\lambda_{2}+\lambda M_{X}\left(\nu e^{r(T-t)}\right)\right) \mu_{21} .
$$

Furthermore, $\hat{g}_{1}(n)$ is an increasing linear function with $\hat{g}_{1}(0)=a$.

Let

$$
t_{2}=T-\frac{1}{r} \ln \left(\frac{1}{\nu} \cdot M_{X}^{-1}\left(-\frac{a+\lambda_{2} \mu_{21}}{\lambda \mu_{21}}\right)\right)=T-\frac{1}{r} \ln \left(\frac{m_{1}}{\nu}\right) .
$$

It is not difficult to see that

$$
a=-\left(\lambda_{2}+\lambda M_{X}\left(\nu e^{r\left(T-t_{2}\right)}\right)\right) \mu_{21}
$$

Then we have the following inequality

$$
\hat{g}_{1}(0)=a<\hat{g}_{2}(0)=-\left(\lambda_{2}+\lambda M_{X}\left(\nu e^{r(T-t)}\right)\right) \mu_{21},
$$

for $0<t<t_{2}$, and thus $\hat{g}_{1}(n)$ and $\hat{g}_{2}(n)$ have a unique point of intersection at some $\hat{n}_{1}(T-t)>0$. For $t>t_{2}$, we obtain

$$
\hat{g}_{1}(0)=a>\hat{g}_{2}(0)=-\left(\lambda_{2}+\lambda M_{X}\left(\nu e^{r(T-t)}\right)\right) \mu_{21},
$$

so $\hat{g}_{1}(n)$ and $\hat{g}_{2}(n)$ have a unique point of intersection at some $\hat{n}_{2}(T-t)<0$. That is, equation (3.11) has a unique root.

Let $t_{1}=T-(1 / r) \ln (\bar{m} / \nu)$ for $\nu<\bar{m}<\nu e^{r T}$. When $\bar{m} \leq \nu$, we denote $t_{1}=T$; and when $\bar{m} \geq \nu e^{r T}$, we denote $t_{1}=0$. Under the constraints of the retention level $q_{t} \in[0,1]$ and investment strategy $u_{t} \geq 0$, we discuss the optimal values in details in the following context.

When $0 \leq t \leq t_{1}$, we have $\left(u^{*}, q^{*}\right)=(\bar{u}(T-t), \bar{q}(T-t))$. Substituting $(\bar{u}(T-t), \bar{q}(T-t))$ into (3.3), we get

$$
h_{1}(T-t)=\tilde{h}_{1}(T-t)+\bar{C},
$$

where

$$
\begin{aligned}
\tilde{h}_{1}(T-t)= & \frac{1}{r}\left[(1+\eta) a_{1}-c\right] \nu e^{r(T-t)}-\left(\lambda_{1}+\lambda_{2}+\lambda+(1+\eta) a_{1} \bar{m}-a \bar{n}\right)(T-t) \\
& +\left(\lambda_{1} M_{X}(\bar{m})+\lambda_{2} M_{Y}(\bar{n})+\lambda M_{X}(\bar{m}) M_{Y}(\bar{n})\right)(T-t)
\end{aligned}
$$

and $\bar{C}$ is a constant that will be determined later.

When $t \geq t_{1}$, we have $\bar{q}(T-t) \geq 1$, and thus $q^{*}=1$. Substituting $q^{*}=1$ into (3.3), we get

$$
\begin{aligned}
\inf _{u}\{ & -h^{\prime}(T-t)-(u a+c) \nu e^{r(T-t)}+\frac{1}{2} u^{2} \sigma^{2} \nu^{2} e^{2 r(T-t)} \\
& +\lambda_{1}\left[M_{X}\left(\nu e^{r(T-t)}\right)-1\right]+\lambda_{2}\left[M_{Y}\left(-\nu u e^{r(T-t)}\right)-1\right] \\
& \left.+\lambda\left[M_{X}\left(\nu e^{r(T-t)}\right) \cdot M_{Y}\left(-\nu u e^{r(T-t)}\right)-1\right]\right\}=0 .
\end{aligned}
$$


Since function $f_{1}(n)$ is an increasing function w.r.t $n \leq n_{0}$, it is not difficult to prove that $\bar{m}<m_{1}$, and thus we have $t_{1}>t_{2}$. By Lemma 3.6, we obtain the minimizer of equation (3.13) given by

$$
\hat{u}(T-t)=-\frac{\hat{n}_{2}(T-t)}{\nu} e^{-r(T-t)},
$$

where $\hat{n}_{2}(T-t)$ is the unique negative root of equation (3.11) for any $t_{1} \leq t \leq T$.

Substituting $(\hat{u}(T-t), 1)$ into $(3.3)$, we get

$$
\begin{aligned}
h_{2}(T-t)= & -\frac{1}{r} c \nu\left(e^{r(T-t)}-1\right)-\left(\lambda_{1}+\lambda_{2}+\lambda\right)(T-t)+\int_{0}^{T-t}\left(a \hat{n}(s)+\frac{1}{2} \sigma^{2} \hat{n}_{2}^{2}(s)\right) d s \\
& +\int_{0}^{T-t}\left(\lambda_{1} M_{X}\left(\nu e^{r s}\right)+M_{Y}\left(\hat{n}_{2}(s)\right)\left(\lambda_{2}+\lambda M_{X}\left(\nu e^{r s}\right)\right)\right) d s .
\end{aligned}
$$

Let

$$
\bar{C}=h_{2}\left(T-t_{1}\right)-\tilde{h}_{1}\left(T-t_{1}\right) .
$$

Then we have

$$
h_{2}\left(T-t_{1}\right)=\tilde{h}_{1}\left(T-t_{1}\right)+\bar{C}=h_{1}\left(T-t_{1}\right) .
$$

The main result of this subsection is summarized in the following theorem.

Theorem 3.1. Recall $(\bar{u}(T-t), \bar{q}(T-t))$ of (3.10) and $\hat{u}(T-t)$ of (3.14). For any $t \in[0, T]$, the optimal strategy for risk model (2.3) is

$$
\left(u^{*}(T-t), q^{*}(T-t)\right)= \begin{cases}(\bar{u}(T-t), \bar{q}(T-t)), & 0 \leq t<t_{1}, \\ (\hat{u}(T-t), 1), & t_{1} \leq t \leq T,\end{cases}
$$

and the value function is

$$
V(t, x)= \begin{cases}-\frac{\beta}{\nu} \exp \left\{-\nu x e^{r(T-t)}+h_{1}(T-t)\right\}, & 0 \leq t \leq t_{1}, \\ -\frac{\beta}{\nu} \exp \left\{-\nu x e^{r(T-t)}+h_{2}(T-t)\right\}, & t_{1} \leq t \leq T .\end{cases}
$$

where $h_{1}(T-t)$ and $h_{2}(T-t)$ are defined in (3.12) and (3.15), respectively.

Remark 3.1. Since

$$
h_{1}\left(T-t_{1}\right)=h_{2}\left(T-t_{1}\right),
$$

$V(t, x)$ is a continuous function for any $(t, x) \in[0, T] \times \mathbb{R}$. Furthermore,

$$
\begin{aligned}
h_{1}^{\prime}\left(T-t_{1}\right)= & -c \bar{m}-a \bar{n}-\left(\lambda_{1}+\lambda_{2}+\lambda\right)+\frac{1}{2} \sigma^{2} \bar{n}^{2} \\
& +\lambda_{1} M_{1}(\bar{n})+M_{2}(\bar{m})\left(\lambda_{2}+\lambda M_{1}(\bar{n})\right) \\
= & h_{2}^{\prime}\left(T-t_{1}\right) .
\end{aligned}
$$

Therefore, we have $V(t, x) \in C^{1,2}$. That is, $V(t, x)$ is a classical solution to the HJB equation (2.5). 


\section{$3.2 \quad$ Variance premium principle}

In this subsection, we assume that the reinsurance premium is calculated according to the variance premium principle. That is,

$$
\delta(q)=(1-q) a_{1}+\Lambda(1-q)^{2} \sigma_{1}^{2},
$$

where $\Lambda$ is the safety loading of reinsurer and $\sigma_{1}^{2}=\left(\lambda_{1}+\lambda\right) \mu_{12}$. Then equation (3.4) becomes

$$
\left\{\begin{array}{l}
-a(t)+u \sigma^{2}(t) \nu e^{r(T-t)}-M_{Y}^{\prime}\left(-\nu u e^{r(T-t)}\right)\left(\lambda_{2}+\lambda M_{X}\left(\nu q e^{r(T-t)}\right)\right)=0, \\
-a_{1}-2 \Lambda \sigma_{1}^{2}+2 \Lambda \sigma_{1}^{2} q+M_{X}^{\prime}\left(\nu q e^{r(T-t)}\right)\left(\lambda_{1}+\lambda M_{Y}\left(-\nu u e^{r(T-t)}\right)\right)=0,
\end{array}\right.
$$

or equivalently,

$$
\left\{\begin{array}{l}
a(t)+\sigma^{2}(t) n+M_{Y}^{\prime}(n)\left(\lambda_{2}+\lambda M_{X}(m)\right)=0, \\
-a_{1}-2 \Lambda \sigma_{1}^{2}+2 \Lambda \sigma_{1}^{2} \frac{m}{\nu} e^{-r(T-t)}+M_{X}^{\prime}(m)\left(\lambda_{1}+\lambda M_{Y}(n)\right)=0,
\end{array}\right.
$$

where $n=-\nu u e^{r(T-t)}$ and $m=\nu q e^{r(T-t)}$. Then we have

Lemma 3.7. For any $t \in[0, T]$, there is a unique positive solution $m_{3}(t)<\nu e^{r(T-t)}$ to the equation

$$
-a_{1}-2 \Lambda \sigma_{1}^{2}+2 \Lambda \sigma_{1}^{2} \frac{m}{\nu} e^{-r(T-t)}+M_{X}^{\prime}(m)\left(\lambda_{1}+\lambda\right)=0 .
$$

Moreover, there exists a unique negative solution $n_{3}<0$ to the equation

$$
-a_{1}-2 \Lambda \sigma_{1}^{2}+\mu_{11}\left(\lambda_{1}+\lambda M_{Y}(n)\right)=0 .
$$

Proof. We first discuss equation (3.17). Let

$$
g_{3}(m)=a_{1}+2 \Lambda \sigma_{1}^{2}-2 \Lambda \sigma_{1}^{2} \frac{m}{\nu} e^{-r(T-t)},
$$

and

$$
g_{4}(m)=M_{X}^{\prime}(m)\left(\lambda_{1}+\lambda\right)
$$

Then we have

$$
\left\{\begin{array}{l}
g_{4}(0)=\left(\lambda_{1}+\lambda\right) \mu_{11}=a_{1}, \\
g_{4}^{\prime}(m)=\left(\lambda_{1}+\lambda\right) M_{X}^{\prime \prime}(m)=\left(\lambda_{1}+\lambda\right) E\left(X^{2} e^{X m}\right)>0, \\
\lim _{m \rightarrow+\infty} g_{4}(m)=+\infty .
\end{array}\right.
$$

Besides, it is easy to see that $g_{3}(m)$ is a decreasing function w.r.t. $m$ with

$$
\left\{\begin{array}{l}
g_{3}(0)=a_{1}+2 \Lambda \sigma_{1}^{2}>g_{4}(0)=a_{1} \\
g_{3}\left(\nu e^{r(T-t)}\right)=a_{1}=g_{4}(0)
\end{array}\right.
$$


Therefore, $g_{3}(m)$ and $g_{4}(m)$ have a unique point of intersection at some point $m_{3}(t) \in\left(0, \nu e^{r(T-t)}\right)$. That is, equation (3.17) has a unique positive root which is less than $\nu e^{r(T-t)}$.

We now return to equation (3.18), which can be rewritten as

$$
M_{Y}(n)=1+\frac{2 \Lambda \sigma_{1}^{2}}{\lambda \mu_{11}}
$$

Using arguments similar to those in the proof of Lemma 3.4, we can prove that (3.19) has a unique negative root. Hence, the proof is completed.

Analogous to Lemma 3.5, one can use Lemma 3.3 and Lemma 3.7 to derive the following inequalities for the existence and uniqueness of the solution to equation (3.16) under the variance premium principle.

Lemma 3.8. Let $m_{1}(t)$ and $m_{3}(t)$ be the unique positive roots of equations (3.6) and (3.17), respectively, and $n_{1}(t)$ and $n_{3}$ be the unique negative roots of equations (3.7) and (3.18), respectively. If inequalities

or

$$
\left\{\begin{array}{l}
m_{1}(t) \quad>m_{3}(t), \\
n_{1}(t) \quad>n_{3}
\end{array}\right.
$$

$$
\left\{\begin{aligned}
m_{1}(t) & <m_{3}(t), \\
n_{1}(t) & <n_{3}
\end{aligned}\right.
$$

hold for any $t \in[0, T]$, then equation (3.16) has a unique root $(\tilde{n}(T-t), \tilde{m}(T-t))$ with $\tilde{n}(T-t)<0$ and $0<\tilde{m}(T-t))<\nu e^{r(T-t)}$.

From Lemma 3.8, we get $-\nu \bar{u}(T-t) e^{r(T-t)}=\tilde{n}(T-t)$ and $\nu \bar{q}(T-t) e^{r(T-t)}=\tilde{m}(T-t)$. Thus, the unique solution to equation (3.2) is given by

$$
\left\{\begin{array}{l}
\bar{u}(T-t)=-\frac{\tilde{n}(T-t)}{\nu} e^{-r(T-t)}, \\
\bar{q}(T-t)=\frac{\tilde{m}(T-t)}{\nu} e^{-r(T-t)} .
\end{array}\right.
$$

Notice that $\tilde{n}(T-t)<0$ and $0<\tilde{m}(T-t))<\nu e^{r(T-t)}$, which imply that $\tilde{u}(T-t)>0$ and $0<\tilde{q}(T-t)<1$. Therefore, we have $\left(u^{*}(T-t), q^{*}(T-t)\right)=(\bar{u}(T-t), \bar{q}(T-t))$. Substituting $\left(u^{*}(T-t), q^{*}(T-t)\right)$ into (3.3), we get

$$
\begin{aligned}
h_{3}(T-t)= & \frac{1}{r}\left(a_{1}-c\right) \nu\left(e^{r(T-t)}-1\right)-\left(\lambda_{1}+\lambda_{2}+\lambda\right)(T-t)+\int_{0}^{T-t} \Lambda\left(1-q^{*}(s)\right)^{2} \sigma_{1}^{2} \nu e^{r s} d s \\
& +\int_{0}^{T-t} a(T-s) \tilde{n}(s)-a_{1} \tilde{m}(s)+\frac{1}{2} \sigma^{2}(T-s) \tilde{n}^{2}(s) d s \\
& +\int_{0}^{T-t} \lambda_{1} M_{X}(\tilde{m}(s))+M_{Y}(\tilde{n}(s))\left(\lambda_{2}+\lambda M_{X}(\tilde{m}(s)) d s .\right.
\end{aligned}
$$

The following theorem summarizes the main result of this subsection. 
Theorem 3.2. Let $(\tilde{n}(T-t), \tilde{m}(T-t))$ be the unique root of equation (3.16). Then, for any $t \in[0, T]$, the optimal strategy for risk model (2.3) under the variance premium principle is

$$
\left\{\begin{array}{l}
u^{*}(T-t)=-\frac{\tilde{n}(T-t)}{\nu} e^{-r(T-t)}, \\
q^{*}(T-t)=\frac{\tilde{m}(T-t)}{\nu} e^{-r(T-t)},
\end{array}\right.
$$

and the value function is given by

$$
V(t, x)=-\frac{\beta}{\nu} \exp \left\{-\nu x e^{r(T-t)}+h_{3}(T-t)\right\}
$$

where $h_{3}(T-t)$ is defined in $(3.20)$.

Remark 3.2. When the risky asset has only negative jumps as in Alvarez E et al. (2014), it is not difficult to see that $M_{Y}(n)$ is a strictly decreasing function w.r.t. $n$. Therefore, we can directly get unique negative solutions $n_{2}$ and $n_{3}$ to the equations

$$
M_{Y}(n)=1+\frac{\lambda_{1}+\lambda}{\lambda} \cdot \eta
$$

and

$$
M_{Y}(n)=1+\frac{2 \Lambda \sigma_{1}^{2}}{\lambda \mu_{11}}
$$

respectively. That is,

$$
n_{2}=M_{Y}^{-1}\left(1+\frac{\lambda_{1}+\lambda}{\lambda} \cdot \eta\right)
$$

and

$$
n_{3}=M_{Y}^{-1}\left(1+\frac{2 \Lambda \sigma_{1}^{2}}{\lambda \mu_{11}}\right) .
$$

Furthermore, the functions $f_{1}(n)$ and $f_{2}(n)$ in the proof of Lemma 3.5 are increasing function w.r.t. $n \in \mathbb{R}$. In this case, the inequalities for the existence and uniqueness of the optimal strategy are easier to derive.

\section{Effects of common shock on the optimal strategy}

In this section, we investigate the effect of the common shock $\lambda$ on the optimal strategy. We begin by considering the case under the expected value principle. For notational convenience, we denote

$$
\left\{\begin{array}{l}
A_{1}=\sigma^{2}(t)+M_{Y}^{\prime \prime}\left(-\nu u e^{r(T-t)}\right)\left(\lambda_{2}+\lambda M_{X}\left(\nu q e^{r(T-t)}\right)\right) \\
B_{1}=M_{X}^{\prime \prime}\left(\nu q e^{r(T-t)}\right)\left(\lambda_{1}+\lambda M_{Y}\left(u \nu e^{r(T-t)}\right)\right) \\
C_{1}=\left(M_{X}^{\prime}\left(\nu q e^{r(T-t)}\right) M_{Y}\left(\nu u e^{r(T-t)}\right)-\mu_{11}\right)
\end{array}\right.
$$


Proposition 4.1. Suppose that the conditions in Lemma 3.5 hold. Then, for any $t \in[0, T]$,

1. $u^{*}(T-t)$ decreases w.r.t. the common shock parameter $\lambda$; and

2. $q^{*}(T-t)$ decreases w.r.t. the parameter $\lambda$ for

$$
0<\eta<\frac{M_{X}^{\prime}\left(\nu q e^{r(T-t)}\right)\left[M_{Y}\left(\nu u e^{r(T-t)}\right)-\frac{\lambda M_{X}\left(\nu q e^{r(T-t)}\right)\left(M_{Y}^{\prime}\left(\nu u e^{r(T-t)}\right)\right)^{2}}{A_{1}}\right]}{\mu_{11}}-1 ;
$$

and increases w.r.t. the parameter $\lambda$ for

$$
\eta>\frac{M_{X}^{\prime}\left(\nu q e^{r(T-t)}\right)\left[M_{Y}\left(\nu u e^{r(T-t)}\right)-\frac{\lambda M_{X}\left(\nu q e^{r(T-t)}\right)\left(M_{Y}^{\prime}\left(\nu u e^{r(T-t)}\right)\right)^{2}}{A_{1}}\right]}{\mu_{11}}-1 .
$$

Proof. If $q^{*}=1$, then the optimal investment strategy $u^{*}(T-t)=\hat{u}(T-t)$ which is the unique solution to the equation

$$
-a(t)+\sigma^{2}(t) u \nu e^{r(T-t)}-M_{Y}^{\prime}\left(-\nu u e^{r(T-t)}\right)\left(\lambda_{2}+\lambda M_{X}\left(\nu e^{r(T-t)}\right)\right)=0 .
$$

Differentiating both sides of this equation w.r.t. $\lambda$ yields

$$
\begin{aligned}
& \sigma^{2}(t) \nu e^{r(T-t)} u^{\prime}(\lambda)-M_{Y}^{\prime}\left(-u \nu e^{r(T-t)}\right) M_{X}\left(\nu e^{r(T-t)}\right) \\
+ & M_{Y}^{\prime \prime}\left(-u \nu e^{r(T-t)}\right)\left(\nu e^{r(T-t)}\right) u^{\prime}(\lambda)\left(\lambda_{2}+\lambda M_{X}\left(\nu e^{r(T-t)}\right)\right)=0,
\end{aligned}
$$

and thus

$$
u^{\prime}(\lambda)=\frac{M_{Y}^{\prime}\left(-u \nu e^{r(T-t)}\right) M_{X}\left(\nu e^{r(T-t)}\right)}{A_{1} \cdot \nu e^{r(T-t)}} .
$$

Since $u^{*}(T-t)>0$, we have $M_{Y}^{\prime}\left(-u^{*}(T-t) \nu e^{r(T-t)}\right)<0$ which yields $u^{\prime}(\lambda)<0$.

If $q^{*}(T-t)=\bar{q}(T-t)$, then $u^{*}(T-t)=\bar{u}(T-t)$. Note that $(\bar{q}(T-t), \bar{u}(T-t))$ is given in (3.10), which is the unique positive solution to the equations

$$
\left\{\begin{array}{l}
-a(t)+u \sigma^{2}(t) \nu e^{r(T-t)}-M_{Y}^{\prime}\left(-\nu u e^{r(T-t)}\right)\left(\lambda_{2}+\lambda M_{X}\left(\nu q e^{r(T-t)}\right)\right)=0, \\
-(1+\eta) a_{1}+M_{X}^{\prime}\left(\nu q e^{r(T-t)}\right)\left(\lambda_{1}+\lambda M_{Y}\left(-\nu u e^{r(T-t)}\right)\right)=0 .
\end{array}\right.
$$

Differentiating both sides of the equations w.r.t. $\lambda$ yields

$$
\left\{\begin{array}{l}
u^{\prime}(\lambda) \sigma^{2}(t) \nu e^{r(T-t)}-M_{Y}^{\prime \prime}\left(-\nu u e^{r(T-t)}\right)\left(-\nu e^{r(T-t)}\right) u^{\prime}(\lambda)\left(\lambda_{2}+\lambda M_{X}\left(\nu q e^{r(T-t)}\right)\right) \\
-M_{Y}^{\prime}\left(-u \nu e^{r(T-t)}\right)\left[M_{X}\left(\nu q e^{r(T-t)}\right)+\lambda M_{X}^{\prime}\left(\nu q e^{r(T-t)}\right)\left(\nu e^{r(T-t)}\right) q^{\prime}(\lambda)\right]=0, \\
M_{X}^{\prime \prime}\left(\nu q e^{r(T-t)}\right)\left(\nu e^{r(T-t)}\right) q^{\prime}(\lambda)\left(\lambda_{1}+\lambda M_{Y}\left(-\nu u e^{r(T-t)}\right)\right) \\
+M_{X}^{\prime}\left(\nu q e^{r(T-t)}\right)\left[M_{Y}\left(-u \nu e^{r(T-t)}\right)+\lambda M_{Y}^{\prime}\left(-\nu u e^{r(T-t)}\right)\left(-\nu e^{r(T-t)}\right) u^{\prime}(\lambda)\right]=0,
\end{array}\right.
$$


and then we get

$$
u^{\prime}(\lambda)=\frac{M_{Y}^{\prime}\left(\nu u e^{r(T-t)}\right)\left[M_{X}\left(\nu q e^{r(T-t)}\right)-\frac{\lambda M_{X}^{\prime}\left(\nu q e^{r(T-t)}\right)\left(C_{1}-\eta \mu_{11}\right)}{B_{1}}\right]}{\left[A_{1}-\frac{\left(\lambda M_{X}^{\prime}\left(\nu q e^{r(T-t)}\right) M_{Y}^{\prime}\left(\nu u e^{r(T-t)}\right)\right)^{2}}{B_{1}}\right] \nu e^{r(T-t)}},
$$

and

$$
q^{\prime}(\lambda)=\frac{(1+\eta) \mu_{11}-M_{X}^{\prime}\left(\nu q e^{r(T-t)}\right)\left[M_{Y}\left(\nu u e^{r(T-t)}\right)-\frac{\lambda M_{X}\left(\nu q e^{r(T-t)}\right)\left(M_{Y}^{\prime}\left(\nu u e^{r(T-t)}\right)\right)^{2}}{A_{1}}\right]}{\left[B_{1}-\frac{\left(\lambda M_{X}^{\prime}\left(\nu q e^{r(T-t)}\right) M_{Y}^{\prime}\left(\nu u e^{r(T-t)}\right)\right)^{2}}{A_{1}}\right] \nu e^{r(T-t)}} .
$$

By Cauchy-Schwarz inequality, it is not difficult to prove that

$$
\left\{\begin{array}{l}
M_{X}\left(\nu q e^{r(T-t)}\right)-\frac{\lambda\left(M_{X}^{\prime}\left(\nu q e^{r(T-t)}\right)\right)^{2} M_{Y}\left(\nu u e^{r(T-t)}\right)}{B_{1}}>0 \\
A_{1}-\sigma^{2}(t)-\frac{\left(\lambda M_{X}^{\prime}\left(\nu q e^{r(T-t)}\right) M_{Y}^{\prime}\left(\nu u e^{r(T-t)}\right)\right)^{2}}{B_{1}}>0 \\
M_{Y}\left(\nu u e^{r(T-t)}\right)-\frac{\lambda M_{X}\left(\nu q e^{r(T-t)}\right)\left(M_{Y}^{\prime}\left(\nu u e^{r(T-t)}\right)\right)^{2}}{A_{1}}>0 \\
B_{1}-\frac{\left(\lambda M_{X}^{\prime}\left(\nu q e^{r(T-t)}\right) M_{Y}^{\prime}\left(\nu u e^{r(T-t)}\right)\right)^{2}}{A_{1}}>0 .
\end{array}\right.
$$

Since $u^{*}(T-t)$ is positive, we can show that $M_{Y}^{\prime}\left(-\nu u^{*}(T-t) e^{r(T-t)}\right)<0$ for any $t \in[0, T]$, and thus $u^{\prime}(\lambda)<0$. As for $q^{\prime}(\lambda)$, it is not difficult to see that its sign strongly depends on the value of $\eta$ and other model parameters.

As for the effect of the common shock $\lambda$ on the optimal strategy under the variance premium principle, we have the following result.

Proposition 4.2. Suppose that the conditions in Lemma 3.8 hold. Then, for any $t \in[0, T]$,

1. $u^{*}(T-t)$ decreases w.r.t. the common shock parameter $\lambda$; and

2. $q^{*}(T-t)$ decreases w.r.t. the parameter $\lambda$ for

$$
0<\Lambda<\frac{M_{X}^{\prime}\left(\nu q e^{r(T-t)}\right)\left[M_{Y}\left(\nu u e^{r(T-t)}\right)-\frac{\lambda M_{X}\left(\nu q e^{r(T-t)}\right)\left(M_{Y}^{\prime}\left(\nu u e^{r(T-t)}\right)\right)^{2}}{A_{1}}\right]-\mu_{11}}{2 \mu_{12}} ;
$$

and increases w.r.t. the parameter $\lambda$ for

$$
\Lambda>\frac{M_{X}^{\prime}\left(\nu q e^{r(T-t)}\right)\left[M_{Y}\left(\nu u e^{r(T-t)}\right)-\frac{\lambda M_{X}\left(\nu q e^{r(T-t)}\right)\left(M_{Y}^{\prime}\left(\nu u e^{r(T-t)}\right)\right)^{2}}{A_{1}}\right]-\mu_{11}}{2 \mu_{12}} .
$$


Proof. Under the variance premium principle, the optimal strategy $\left(u^{*}(T-t), q^{*}(T-t)\right)=$ $(\bar{u}(T-t), \bar{q}(T-t))$ is the unique positive solution to the equation

$$
\left\{\begin{array}{l}
-a(t)+u \sigma^{2}(t) \nu e^{r(T-t)}-M_{Y}^{\prime}\left(-\nu u e^{r(T-t)}\right)\left(\lambda_{2}+\lambda M_{X}\left(\nu q e^{r(T-t)}\right)\right)=0, \\
-a_{1}-2 \Lambda \sigma_{1}^{2}+2 \Lambda \sigma_{1}^{2} q+M_{X}^{\prime}\left(\nu q e^{r(T-t)}\right)\left(\lambda_{1}+\lambda M_{Y}\left(-\nu u e^{r(T-t)}\right)\right)=0 .
\end{array}\right.
$$

Similar to the proof of Proposition 4.1, one can also get

$$
u^{\prime}(\lambda)=\frac{M_{Y}^{\prime}\left(\nu u e^{r(T-t)}\right)\left[M_{X}\left(\nu q e^{r(T-t)}\right)-\frac{\lambda M_{X}^{\prime}\left(\nu q e^{r(T-t)}\right)\left(C_{1}-2 \Lambda \mu_{12}\right) \nu e^{r(T-t)}}{2 \Lambda \sigma_{1}^{2}+B_{1} \cdot \nu e^{r(T-t)}}\right] e^{-r(T-t)}}{\left[A_{1}-\frac{\left(\lambda M_{X}^{\prime}\left(\nu q e^{r(T-t)}\right) M_{Y}^{\prime}\left(\nu u e^{r(T-t)}\right)\right)^{2} \nu e^{r(T-t)}}{2 \Lambda \sigma_{1}^{2}+B_{1} \cdot \nu e^{r(T-t)}}\right] \nu},
$$

and

$$
q^{\prime}(\lambda)=\frac{\mu_{11}+2 \Lambda \mu_{12}-M_{X}^{\prime}\left(\nu q e^{r(T-t)}\right)\left[M_{Y}\left(\nu u e^{r(T-t)}\right)-\frac{\lambda M_{X}\left(\nu q e^{r(T-t)}\right)\left(M_{Y}^{\prime}\left(\nu u e^{r(T-t)}\right)\right)^{2}}{A_{1}}\right]}{2 \Lambda \sigma_{1}^{2}+B_{1} \cdot \nu e^{r(T-t)}-\frac{\left(\lambda M_{X}^{\prime}\left(\nu q e^{r(T-t)}\right) M_{Y}^{\prime}\left(\nu u e^{r(T-t)}\right)\right)^{2} \nu e^{r(T-t)}}{A_{1}}} .
$$

Again, by applying the Cauchy-Schwarz inequality and making use of (4.1), one can obtain the desired results.

Remark 4.1. From the results in Proposition 4.1 and Proposition 4.2, we see that the optimal investment strategy $u^{*}(T-t)$ with common shock is always smaller than the one without common shock dependence not only under the expected value principle but also under the variance premium principle. For the optimal reinsurance strategy $q^{*}(T-t)$, whether the value with common shock is smaller or larger than that without common shock strongly depends on the values of the parameters in the model of study.

\section{$5 \quad$ Effects of $\lambda_{1}, \lambda_{2}$ and $\nu$}

In this section, we continue to investigate the effect of other model parameters, namely $\lambda_{1}, \lambda_{2}$ and $\nu$, on the optimal strategy. Again, we begin by considering the case under the expected value principle.

Proposition 5.1. Suppose that the conditions in Lemma 3.5 hold. Then

1. If full retention is optimal, i.e., $q^{*}(T-t) \equiv 1$, the optimal investment strategy $u^{*}(T-t)$ is independent with parameter $\lambda_{1}$, and decreases with respect to parameter $\lambda_{2}$;

2. If partial retention is optimal, i.e., $q^{*}(T-t)=\bar{q}(T-t)$, then for any $t \in[0, T], u^{*}(T-t)$ decreases w.r.t. each of $\lambda_{1}$ and $\lambda_{2}$; while $q^{*}(T-t)$ increases w.r.t. each of $\lambda_{1}$ and $\lambda_{2}$.

Proof. When full retention level is optimal, i.e., $q^{*}=1$, then for any $t \in[0, T]$, the optimal investment strategy $u^{*}(T-t)=\hat{u}(T-t)$ is the unique positive solution to the equation

$$
-a(t)+\sigma^{2}(t) u \nu e^{r(T-t)}-M_{Y}^{\prime}\left(-\nu u e^{r(T-t)}\right)\left(\lambda_{2}+\lambda M_{X}\left(\nu e^{r(T-t)}\right)\right)=0,
$$


Differentiating both sides of this equation w.r.t. $\lambda_{2}$ yields

$$
u^{\prime}\left(\lambda_{2}\right)=\frac{M_{Y}^{\prime}\left(-u \nu e^{r(T-t)}\right) e^{-r(T-t)}}{A_{1} \cdot \nu} .
$$

Since $u^{*}(T-t)=\hat{u}(T-t)$ is positive, it is not difficult to see that $M_{Y}^{\prime}\left(-u^{*}(T-t) \nu e^{r(T-t)}\right)<0$, and thus $u^{\prime}\left(\lambda_{2}\right)<0$.

When partial retention is optimal, we have $q^{*}(T-t)=\bar{q}(T-t)$ and $u^{*}(T-t)=\bar{u}(T-t)$ where $(\bar{q}(T-t), \bar{u}(T-t))$ is the unique positive solution to the equation

$$
\left\{\begin{array}{l}
-a(t)+u \sigma^{2}(t) \nu e^{r(T-t)}-M_{Y}^{\prime}\left(-\nu u e^{r(T-t)}\right)\left(\lambda_{2}+\lambda M_{X}\left(\nu q e^{r(T-t)}\right)\right)=0, \\
-(1+\eta)\left(\lambda_{1}+\lambda\right) \mu_{11}+M_{X}^{\prime}\left(\nu q e^{r(T-t)}\right)\left(\lambda_{1}+\lambda M_{Y}\left(-\nu u e^{r(T-t)}\right)\right)=0 .
\end{array}\right.
$$

Differentiating both sides of equations w.r.t. each of $\lambda_{1}$ and $\lambda_{2}$ gives

$$
\left\{\begin{array}{l}
q^{\prime}\left(\lambda_{1}\right)=\frac{-M_{X}^{\prime}\left(\nu q e^{r(T-t)}\right)+(1+\eta) \mu_{11}}{\left(B_{1}-\frac{\left(\lambda M_{X}^{\prime}\left(\nu q e^{r(T-t)}\right) M_{Y}^{\prime}\left(-\nu u e^{r(T-t)}\right)\right)^{2}}{A_{1}}\right) \nu e^{r(T-t)}}, \\
q^{\prime}\left(\lambda_{2}\right)=\frac{\lambda M_{X}^{\prime}\left(\nu q e^{r(T-t)}\right)\left(M_{Y}^{\prime}\left(-\nu u e^{r(T-t)}\right)\right)^{2}}{\left(A_{1} \cdot B_{1}-\left(\lambda M_{X}^{\prime}\left(\nu q e^{r(T-t)}\right) M_{Y}^{\prime}\left(-\nu u e^{r(T-t)}\right)\right)^{2}\right) \nu e^{r(T-t)}}, \\
u^{\prime}\left(\lambda_{1}\right)=\frac{\lambda M_{X}^{\prime}\left(\nu q e^{r(T-t)}\right) M_{Y}^{\prime}\left(-\nu u e^{r(T-t)}\right)\left(-M_{X}^{\prime}\left(\nu q e^{r(T-t)}\right)+(1+\eta) \mu_{11}\right)}{\left(A_{1} \cdot B_{1}-\left(\lambda M_{X}^{\prime}\left(\nu q e^{r(T-t)}\right) M_{Y}^{\prime}\left(-\nu u e^{r(T-t)}\right)\right)^{2}\right) \nu e^{r(T-t)}}, \\
u^{\prime}\left(\lambda_{2}\right)=\frac{M_{Y}^{\prime}\left(\nu q e^{r(T-t)}\right)}{\left(A_{1}-\frac{\left(\lambda M_{X}^{\prime}\left(\nu q e^{r(T-t)}\right) M_{Y}^{\prime}\left(-\nu u e^{r(T-t)}\right)\right)^{2}}{B_{1}}\right) \nu e^{r(T-t)}} .
\end{array}\right.
$$

According to (4.1), it is not difficult to prove that $q^{\prime}\left(\lambda_{2}\right)>0$ and $u^{\prime}\left(\lambda_{2}\right)<0$ for any $t \in[0, T]$. For the effect of $\lambda_{1}$, we can see that the values of $q^{\prime}\left(\lambda_{1}\right)$ and $u^{\prime}\left(\lambda_{1}\right)$ strongly depend on the value of $-M_{X}^{\prime}\left(\nu q e^{r(T-t)}\right)+(1+\eta) \mu_{11}$. That is, when $-M_{X}^{\prime}\left(\nu q e^{r(T-t)}\right)+(1+\eta) \mu_{11}>0$ holds, we have $u^{\prime}\left(\lambda_{1}\right)<0$ while $q^{\prime}\left(\lambda_{1}\right)>0$; otherwise, we have $u^{\prime}\left(\lambda_{1}\right)>0$ while $q^{\prime}\left(\lambda_{1}\right)<0$.

It follows from (3.1) that

$$
(1+\eta)\left(\lambda_{1}+\lambda\right) \mu_{11}=M_{X}^{\prime}\left(\nu \bar{q}(T-t) e^{r(T-t)}\right)\left(\lambda_{1}+\lambda M_{Y}\left(-\nu \bar{u}(T-t) e^{r(T-t)}\right)\right) .
$$

From the proof of Lemma 3.4, it is not difficult to see that for any $n<0$, we have $M_{Y}(n)>M_{Y}(0)=$ 1 , and hence $M_{Y}\left(-\nu \bar{u}(T-t) e^{r(T-t)}\right)>1$. Therefore, we obtain

$$
\begin{aligned}
(1+\eta)\left(\lambda_{1}+\lambda\right) \mu_{11} & =M_{X}^{\prime}\left(\nu \bar{q}(T-t) e^{r(T-t)}\right)\left(\lambda_{1}+\lambda M_{Y}\left(-\nu \bar{u}(T-t) e^{r(T-t)}\right)\right), \\
& >M_{X}^{\prime}\left(\nu \bar{q}(T-t) e^{r(T-t)}\right)\left(\lambda_{1}+\lambda\right),
\end{aligned}
$$

which means that inequality

$$
(1+\eta) \mu_{11}>M_{X}^{\prime}\left(\nu \bar{q}(T-t) e^{r(T-t)}\right)
$$


always holds for any $t \in[0, T]$.

To end the section, we present the following proposition which explores the effect of $\lambda_{1}$ and $\lambda_{2}$ on the optimal strategy under the variance premium principle.

Proposition 5.2. Suppose that the conditions in Lemma 3.8 hold. Then, for any $t \in[0, T], u^{*}(T-t)$ decreases w.r.t each of $\lambda_{1}$ and $\lambda_{2}$; while $q^{*}(T-t)$ increases w.r.t. each of $\lambda_{1}$ and $\lambda_{2}$.

In the following proposition, we determine the effect of parameter $\nu$ on the optimal strategy. Here $\nu$ is the constant absolute risk aversion parameter for the decision-maker.

Proposition 5.3. Suppose that the conditions in Lemma 3.5 or Lemma 3.8 hold. Then, for any $t \in[0, T], u^{*}(T-t)$ and $q^{*}(T-t)$ decrease with respect to the risk aversion parameter $\nu$, respectively.

Proof. For the expected value principle, when full retention is optimal, we obtain

$$
u^{\prime}(\nu)=\frac{\lambda M_{Y}^{\prime}\left(-\nu u e^{r(T-t)}\right) M_{X}^{\prime}\left(\nu q e^{r(T-t)}\right)-u \cdot A_{1}}{\nu \cdot A_{1}} .
$$

Since the value of $M_{Y}^{\prime}\left(-\nu u e^{r(T-t)}\right)$ is negative at the optimal investment strategy $u^{*}$, one can show that $u^{\prime}(\nu)<0$. On the other hand, when partial retention is optimal, we have

$$
\left[A_{1}-\frac{\left(\lambda M_{X}^{\prime}\left(\nu q e^{r(T-t)}\right) M_{Y}^{\prime}\left(\nu u e^{r(T-t)}\right)\right)^{2}}{B_{1}}\right] \cdot\left(u^{\prime}(\nu) \nu+u\right)=0 .
$$

Since

$$
A_{1}-\frac{\left(\lambda M_{X}^{\prime}\left(\nu q e^{r(T-t)}\right) M_{Y}^{\prime}\left(\nu u e^{r(T-t)}\right)\right)^{2}}{B_{1}}>0
$$

always holds for any $t \in[0, T]$, we have $u^{\prime}(\nu) \nu+u=0$, which means that $u^{\prime}(\nu)=-u / \nu<0$. Similarly, it can be shown that $q^{\prime}(\nu)=-q / \nu<0$.

For the variance premium principle case, we have

$$
q^{\prime}(\nu)=\frac{-\left[B_{1} \cdot \nu e^{r(T-t)}-\frac{\left(\lambda M_{X}^{\prime}\left(\nu q e^{r(T-t)}\right) M_{Y}^{\prime}\left(\nu u e^{r(T-t)}\right)\right)^{2} \nu e^{r(T-t)}}{A_{1}}\right] q}{2 \Lambda \sigma_{1}^{2}+\left[B_{1} \cdot \nu e^{r(T-t)}-\frac{\left(\lambda M_{X}^{\prime}\left(\nu q e^{r(T-t)}\right) M_{Y}^{\prime}\left(\nu u e^{r(T-t)}\right)\right)^{2} \nu e^{r(T-t)}}{A_{1}}\right] \nu},
$$

and

$$
u^{\prime}(\nu)=\frac{\frac{2 \Lambda \sigma_{1}^{2} q}{\nu}-u\left[\frac{B_{1} \cdot \nu e^{r(T-t)}}{\lambda M_{X}^{\prime}\left(\nu q e^{r(T-t)}\right) M_{Y}^{\prime}\left(\nu u e^{r(T-t)}\right)}-\frac{\lambda M_{X}^{\prime}\left(\nu q e^{r(T-t)}\right) M_{Y}^{\prime}\left(\nu u e^{r(T-t)}\right)}{A_{1}}\right]}{\frac{2 \Lambda \sigma_{1}^{2}+\nu B_{1} \cdot \nu e^{r(T-t)}}{\lambda M_{X}^{\prime}\left(\nu q e^{r(T-t)}\right) M_{Y}^{\prime}\left(\nu u e^{r(T-t)}\right)}-\frac{\lambda M_{X}^{\prime}\left(\nu q e^{r(T-t)}\right) M_{Y}^{\prime}\left(\nu u e^{r(T-t)}\right)}{A_{1}}} .
$$

Since $M_{Y}^{\prime}\left(\nu u e^{r(T-t)}\right)<0$ at $u=u^{*}$, it follows from (4.1) that

$$
\frac{B_{1} \cdot \nu e^{r(T-t)}}{\lambda M_{X}^{\prime}\left(\nu q e^{r(T-t)}\right) M_{Y}^{\prime}\left(\nu u e^{r(T-t)}\right)}-\frac{\lambda M_{X}^{\prime}\left(\nu q e^{r(T-t)}\right) M_{Y}^{\prime}\left(\nu u e^{r(T-t)}\right)}{A_{1}}<0,
$$


and

$$
B_{1} \cdot \nu e^{r(T-t)}-\frac{\left(\lambda M_{X}^{\prime}\left(\nu q e^{r(T-t)}\right) M_{Y}^{\prime}\left(\nu u e^{r(T-t)}\right)\right)^{2} \nu e^{r(T-t)}}{A_{1}}>0,
$$

which implies that $q^{\prime}(\nu)<0$ and $u^{\prime}(\nu)<0$.

\section{$6 \quad$ Numerical examples}

In this section, we again assume that $a(t) \equiv a$ and $\sigma(t) \equiv \sigma$ are constant parameters. Furthermore, we assume that the density functions of claim sizes $\left\{X_{i}\right\}$ and jump amplitudes $\left\{Y_{i}\right\}$ are given by

$$
f_{1}(x)= \begin{cases}\alpha_{1} e^{-\alpha_{1} x}, & x>0, \\ 0, & x<0,\end{cases}
$$

and

$$
f_{2}(y)= \begin{cases}\alpha_{2} e^{\alpha_{2} y}, & y<0 \\ 0, & y>0\end{cases}
$$

respectively, with $\alpha_{1}>0$ and $\alpha_{2}>0$. Then we have

$$
\left\{\begin{aligned}
M_{X}\left(\nu q e^{r(T-t)}\right) & =\frac{\alpha_{1}}{\alpha_{1}-\nu q e^{r(T-t)}}, \\
M_{Y}\left(-\nu u e^{r(T-t)}\right) & =\frac{\alpha_{2}}{\alpha_{2}-\nu u e^{r(T-t)}} .
\end{aligned}\right.
$$

The minimizer $(\bar{q}(T-t), \bar{u}(T-t))$ of $(3.3)$ under the expected value principle satisfies the following equations

$$
\left\{\begin{aligned}
a-u \sigma^{2} \nu e^{r(T-t)} & =\frac{\alpha_{2}}{\left(\alpha_{2}-\nu u e^{r(T-t)}\right)^{2}}\left(\lambda_{2}+\frac{\lambda \alpha_{1}}{\alpha_{1}-\nu q e^{r(T-t)}}\right), \\
\frac{(1+\eta)\left(\lambda_{1}+\lambda\right)}{\alpha_{1}} & =\frac{\alpha_{1}}{\left(\alpha_{1}-\nu q e^{r(T-t)}\right)^{2}}\left(\lambda_{1}+\frac{\lambda \alpha_{2}}{\alpha_{2}-\nu u e^{r(T-t)}}\right),
\end{aligned}\right.
$$

and the minimizer $(\bar{q}(T-t), \bar{u}(T-t))$ of $(3.3)$ under the variance premium principle is the solution to the following equations

$$
\left\{\begin{array}{c}
a-u \sigma^{2} \nu e^{r(T-t)}=\frac{\alpha_{2}}{\left(\alpha_{2}-\nu u e^{r(T-t)}\right)^{2}}\left(\lambda_{2}+\frac{\lambda \alpha_{1}}{\alpha_{1}-\nu q e^{r(T-t)}}\right), \\
a_{1}+2 \Lambda \sigma_{1}^{2}(1-q)=\frac{\alpha_{1}}{\left(\alpha_{1}-\nu q e^{r(T-t)}\right)^{2}}\left(\lambda_{1}+\frac{\lambda \alpha_{2}}{\alpha_{2}-\nu u e^{r(T-t)}}\right),
\end{array}\right.
$$

where $a_{1}=\left(\lambda_{1}+\lambda\right) / \alpha_{1}$ and $\sigma_{1}^{2}=2\left(\lambda_{1}+\lambda\right) / \alpha_{1}^{2}$.

Besides, for the expected value principle, if $q^{*}(T-t) \equiv 1$, then $u^{*}(T-t)=\hat{u}(T-t)$ satisfies the equation

$$
a-u \sigma^{2} \nu e^{r(T-t)}=\frac{\alpha_{2}}{\left(\alpha_{2}-\nu u e^{r(T-t)}\right)^{2}}\left(\lambda_{2}+\frac{\lambda \alpha_{1}}{\alpha_{1}-\nu e^{r(T-t)}}\right) .
$$


In the following two examples, we let $\eta=\Lambda \sigma_{1}^{2} / a_{1}$. Hence, we have

$$
(1+\eta) a_{1}=a_{1}+\Lambda \sigma_{1}^{2},
$$

which implies that when the insurer transfers all the risk to the reinsurer, i.e., $q=0$, the reinsurance premiums under the two premium principles are the same.

Remark 6.1. Note that, under condition (6.1), for any $q \in(0,1)$, we have

$$
\begin{aligned}
(1+\eta)(1-q) a_{1} & =(1-q) a_{1}+\eta(1-q) a_{1} \\
& =(1-q) a_{1}+\Lambda(1-q) \sigma_{1}^{2} \\
& >(1-q) a_{1}+\Lambda(1-q)^{2} \sigma_{1}^{2} .
\end{aligned}
$$

Inequality (6.2) implies that under condition (6.1), the reinsurance premium calculated under expected value principle is more expensive than that under the variance premium principle.

\begin{tabular}{|c|c|c|c|c|c|c|c|c|c|c|}
\hline \multicolumn{2}{|l|}{ ) } & 0 & 0.05 & 0.1 & 0.2 & 0.25 & 0.3 & 0.4 & 0.5 & 0.6 \\
\hline expected value & $q^{*}$ & 0.85450 & 0.83365 & 0.82322 & 0.81799 & 0.81955 & 0.82264 & 0.83155 & 0.84231 & 0.85379 \\
\hline principle & $u^{*}$ & 1.42342 & 1.23783 & 1.07927 & 0.80923 & 0.68982 & 0.57794 & 0.37186 & 0.18406 & 0.01040 \\
\hline variance premium & $q^{*}$ & 0.68130 & 0.67008 & 0.66417 & 0.66047 & 0.66083 & 0.66202 & 0.66586 & 0.67074 & 0.67606 \\
\hline principle & $u^{*}$ & 1.42342 & 1.25882 & 1.11503 & 0.86754 & 0.75793 & 0.65538 & 0.46720 & 0.29674 & 0.14009 \\
\hline
\end{tabular}

Example 6.1. In this example, we set $T=15, t=5, r=0.05, b=0.3, \sigma=0.35, \nu=0.6, \Lambda=2$, $\eta=2, \alpha_{1}=2$ and $\alpha_{2}=5$. The results are shown in Tables 1,2 and 3 .

\begin{tabular}{|c|c|c|c|c|c|c|c|c|c|c|}
\hline$\lambda_{1}$ & & 0.1 & 0.2 & 0.3 & 0.4 & 0.5 & 0.6 & 0.7 & 0.8 & 0.9 \\
\hline expected value & & 0.797 & 0.00 & 0.01 & 0.82264 & 0.82669 & 0.82983 & 0.83233 & 0.83438 & 0.83607 \\
\hline principle & $u^{*}$ & 0.59092 & 0.58487 & 0.58083 & 0.57794 & & 0.57408 & & & 0.57071 \\
\hline um & $q^{*}$ & 0.64766 & 065435 & 065882 & 066202 & 0.66442 & 0.66629 & 0.66779 & 0.66901 & 0.67003 \\
\hline principle & $u^{*}$ & 0.66158 & 0.65871 & 0.65677 & 0.65538 & 0.65433 & 0.65351 & 0.65286 & 0.65231 & 0.65187 \\
\hline
\end{tabular}

Table 1 Effect of $\lambda$ on the optimal strategy

Table 2 Effect of $\lambda_{1}$ on the optimal strategy 
Table 3 Effect of $\lambda_{2}$ on the optimal strategy

\begin{tabular}{|c|c|c|c|c|c|c|c|c|c|c|}
\hline$\lambda_{2}$ & & 0 & 0.05 & 0.1 & 0.2 & 0.3 & 0.4 & 0.5 & 0.6 & 0.7 \\
\hline expected val & & 0.80388 & 0.80916 & 0.81400 & 0.82264 & 0.83016 & 0.8368 & 04078 & 0.07018 & 0.85309 \\
\hline & & & & & & & & & & 0.02840 \\
\hline e premium & & & & & & م & acroen & & & 0.67887 \\
\hline principle & & 0.95335 & 0 . & 0.79666 & & 0.52613 & 0.40660 & & $0.180+1$ & 0.09154 \\
\hline
\end{tabular}

From Table 1 with $\lambda_{1}=0.4$ and $\lambda_{2}=0.2$, we see that the optimal strategy $q^{*}$ decreases with $\lambda \in[0,0.25]$, and then increases with $\lambda$ for $\lambda>0.25$ while $u^{*}$ decreases as the value of $\lambda$ increases. The numerical values in Table 2 with $\lambda_{2}=0.2$ and $\lambda=0.3$ and Table 3 with $\lambda_{1}=0.4$ and $\lambda=0.3$ indicate that a greater value of $\lambda_{1}$ (or $\lambda_{2}$ ) yields a greater value of $q^{*}$ but a smaller value of $u^{*}$. These results are natural consequences of Propositions 4.1, 4.2, 5.1 and 5.2.

When comparing the optimal results between the two premium principles, we find that, under (6.1), the optimal reinsurance strategy under the expected value principle is always larger than the one under the variance premium principle. On the other hand, for $\lambda \neq 0$, the optimal investment strategy under the expected value principle is always less than that under variance premium principle. These results are expected since the variance premium principle is cheaper than the expected value principle under (6.1) (see Remark 6.1). However, for $\lambda=0$, we see from the two bold numbers in Table 1 that the optimal investment strategies are the same under the two premium principles. This suggests that when the dependence between the risky asset and the aggregate claims is vanished, the choice of premium principle do affect the optimal reinsurance strategy but not the optimal investment strategy.

Example 6.2. In this example, we set $T=15, t=5, r=0.05, b=0.3, \sigma=0.35, \Lambda=2, \eta=2$, $\lambda_{1}=0.4, \lambda_{2}=0.2, \lambda=0.3, \alpha_{1}=2$, and $\alpha_{2}=5$. Table 4 presents the impact of $\nu$ on the optimal results.

Table 4 Effect of $\nu$ on the optimal strategy

\begin{tabular}{|c|c|c|c|c|c|c|c|c|c|c|}
\hline \multicolumn{2}{|l|}{$\nu$} & 0 & 0.1 & 0.2 & 0.3 & 0.4 & 0.5 & 0.6 & 0.7 & 0.8 \\
\hline expected value & $q^{*}$ & 1 & 1 & 1 & 1 & 0.98717 & 0.82264 & 0.70512 & 0.61698 & 0.54843 \\
\hline principle & $u^{*}$ & 4.93470 & 2.32594 & 1.43960 & 0.98002 & 0.69353 & 0.57794 & 0.49538 & 0.43346 & 0.38529 \\
\hline ariance premium & $q^{*}$ & 0.93211 & 0.88079 & 0.82575 & 0.76942 & 0.71423 & 0.66202 & & & 0.53087 \\
\hline ртілекіе & $u^{*}$ & 4.95234 & 2.36171 & 1.50000 & 1.07260 & 0.82001 & 0.65538 & 0.54085 & 0.45744 & 0.39450 \\
\hline
\end{tabular}

We see from Table 4 that both $q^{*}$ and $u^{*}$ decrease as $\nu$ increases. Note that $\nu$ is the constant 
absolute risk aversion parameter of the utility function, a large value of $\nu$ means more risk averse. This implies that if the decision maker is more risk averse, a larger portion of the underlying risk will be transferred to a reinsurer and less money will be invested into the risky asset. This is also a natural consequence of Proposition 5.3. Moreover, in Table 4, we see that the optimal results of the two premium principles behave like those observed in Tables $1 \sim 3$.

\section{Conclusion}

We first recap the main results of the paper. From an insurer's point of view, we consider the optimal reinsurance and investment problem with jump-diffusion risky asset in a compound Poisson risk model, where the aggregate claims and the stock price are correlated through a common shock. With some constraints on the control variables, we use a nonstandard approach to investigate the existence and uniqueness of the optimal reinsurance and investment strategy. Under the criterion of maximizing the expected exponential utility, we derive the closed-form expressions for the optimal strategy and value function for two frequently-used premium principles, namely the expected value principle and the variance premium principle. Furthermore, we examine the impact of the common shock parameter as well as some other model parameters on the optimal strategy. The numerical results show that as the degree of common shock dependence increases, the optimal investment strategy decreases but the associated optimal reinsurance strategy does not necessarily decrease. The latter also depends very much on the values of other parameters in the model of study. Besides the common shock parameter, we numerically examine the effects of other model parameters on the optimal strategy which are more or less consistent with the analytical results.

As for future research, there are still other interesting problems in this direction for further investigation. For example, one may consider the optimal reinsurance and investment problem with dependence in the framework of stochastic differential games with partial information, or in a Markovian regime-switching economy. Moreover, additional constraints on the probability of

ruin certainly make the optimal reinsurance and investment problem with the expected utility very challenging, especially for risk processes with jumps.

\section{Acknowledgements}

The research of Zhibin Liang and Caibin Zhang was supported by the National Natural Science Foundation of China (Grant No.11471165) and Jiangsu Natural Science Foundation (Grant No. BK20141442). The research of Kam Chuen Yuen was supported by a grant from the Research Grants Council of the Hong Kong Special Administrative Region, China (Project No. HKU17329216). 


\section{References}

[1] Alvarez E, L. H. R., Matomäki, P. and Rakkolainen, T. A. (2014). A class of solvable optimal stopping problems of spectrally negative jump diffusion. SIAM Journal on Control and Optimization 52(4), 2224-2249.

[2] Bai, L. and Guo, J. (2008). Optimal proportional reinsurance and investment with multiple risky assets and no-shorting constraint. Insurance: Mathematics and Economics 42, 968-975.

[3] Bi, J. and Guo, J. (2013). Optimal mean-variance problem with constrained controls in a jump-diffusion financial market for an insurer. Journal of Optimization Theory and Applications 157, 252-275.

[4] Bi, J., Liang, Z. and Xu, F. (2016). Optimal mean-variance investment and reinsurance problems for the risk model with common shock dependence. Insurance: mathematics and Economics 70, 245-258.

[5] Bernanke, B. S. (1983). Irreversibility, uncertainty, and cyclical investment. Quarterly Journal of Economics 89, 85-103.

[6] Bernard, C. and Tian, W. (2009). Optimal reinsurance arrangements under tail risk measures. Journal of Risk and Insurance 76(3), 709-725.

[7] Browne, S. (1995). Optimal investment policies for a firm with random risk process: exponential utility and minimizing the probability of ruin. Mathematics of Operations Research 20, 937-958.

[8] Cai, J. and Tan, K. (2007). Optimal retention for a stop-loss reinsurance under the VaR and CTE risk measures. ASTIN Bulletin 37(1), 93-112.

[9] Cai, J., Tan, K., Weng, C. and Zhang, Y. (2008). Optimal reinsurance under VaR and CTE risk measures. Insurance: Mathematics and Economics 43, 185-196.

[10] Centeno, M. (1986). Measuring the effects of reinsurance by the adjustment coefficient. Insurance: Mathematics and Economics 5, 169-182.

[11] Centeno, M. (2002). Excess of loss reinsurance and Gerber's inequality in the Sparre Anderson model. Insurance: Mathematics and Economics 31, 415-427.

[12] Fleming, W. and Soner, H. (2006). Controlled Markov Processes and Viscosity Solutions. 2nd Ed. Springer-Verlag, New York.

[13] Gerber, H. (1979). An Introduction to Mathematical Risk Theory. In: S.S. Huebner Foundation Monograph, Series No. 8. Irwin, Homewood, Ill.

[14] Hald, M. and Schmidli, H. (2004). On the maximization of the adjustment coefficient under proportioal reinsurance. ASTIN Bulletin 34(1), 75-83.

[15] Irgens, C. and Paulsen, J. (2004). Optimal control of risk exposure, reinsurance and investments for insurance portfolios. Insurance: Mathematics and Economics 35, 21-51.

[16] Kaluszka, M. (2001). Optimal reinsurance under mean-variance premium principles. Insurance: Mathematics and Economics 28, 61-67.

[17] Kaluszka, M. (2004). Mean-variance optimal reinsurance arrangements. Scandinavian Actuarial Journal 1, 28-41.

[18] Landriault, D., Li, B., Li, D. and Li, D. (2016). A pair of optimal reinsurance-investment strategies in the two-sided exit framework. Insurance: Mathematics and Economics 71, 284-294. 
[19] Liang, Z. (2007). Optimal proportional reinsurance for controlled risk process which is perturbed by diffusion. Acta Mathematicae Applicatae Sinica, English Series 23, 477-488.

[20] Liang, Z. and Bayraktar, E. (2014). Optimal proportional reinsurance and investment with unobservable Markov-modulated compound Poisson process. Insurance: Mathematics and Economics 55, 156-166.

[21] Liang, Z., Bi, J., Yuen, K. C. and Zhang, C. (2016). Optimal mean-variance reinsurance and investment in a jump-diffusion financial market with common shock dependence. Mathematical Methods of Operation Research 84, 155-181.

[22] Liang, Z. and Guo, J. (2007). Optimal proportional reinsurance and ruin probability. Stochastic Models $23,333-350$.

[23] Liang, Z. and Guo, J. (2008). Upper bound for ruin probabilities under optimal investment and proportional reinsurance. Applied Stochastic Models in Business and Industry 24, 109-128.

[24] Liang, Z., Yuen, K. C. and Guo, J. (2011). Optimal proportional reinsurance and investment in a stock market with Ornstein-Uhlenbeck process. Insuarnce: Mathematics and Economics 49, 207-215.

[25] Liang, Z., Yuen, K. C. and Cheung, K. C. (2012). Optimal reinsurance and investment problem in a constant elasticity of variance stock market for jump-diffusion risk model. Applied Stochastic Models in Business and Industry 28, 585-597.

[26] Luo, S., Taksar, M. and Tsoi, A. (2008). On reinsurance and investment for large insurance portfolios. Insurance: Mathematics and Economics 42, 434-444.

[27] Promislow, D. and Young, V. (2005). Minimizing the probability of ruin when claims follow Brownian motion with drift. North American Actuarial Journal 9(3), 109-128.

[28] Schmidli, H. (2001). Optimal proportional reinsurance policies in a dynamic setting. Scandinavian Actuarial Journal 1, 55-68.

[29] Schmidli, H. (2002). On minimizing the ruin probability by investment and reinsurance. Annals of Applied Probability 12, 890-907.

[30] Yang, H. and Zhang, L. (2005). Optimal investment for insurer with jump-diffusion risk process. Insurance: Mathematics and Economics 37, 615-634.

[31] Yuen, K. C., Liang, Z. and Zhou. M. (2015). Optimal proportional reinsurance with common shock dependence. Insurance: Mathematics and Economics 64, 1-13.

[32] Zhang, X., Meng, H. and Zeng, Y. (2016). Optimal investment and reinsurance strategies for insurers with generalized mean-variance premium principle and no-short selling. Insuarnce: Mathematics and Economics 67, 125-132.

[33] Zheng, X., Zhou, J. and Sun, Z. (2016). Robust optimal portfolio and proportional reinsurance for an insurer under a CEV model. Insuarnce: Mathematics and Economics 67, 77-87.

Correspondence author: Dr. Zhibin Liang, School of Mathematical Sciences, Nanjing Normal University, Jiangsu 210023, P.R.China 